\title{
EELS and EFTEM of Surface Plasmons in Metallic Nanostructures
}

W. Sigle, ${ }^{*}$ L. Gu****, N. Talebi,***, B. Ögüt, ${ }^{*}$ C. T. Koch, R. Vogelgesang, ${ }^{* * *}$ and P. A. van Aken*

* Max Planck Institute for Metals Research, Heisenbergstraße 3, D-70569 Stuttgart

** Photonics Research Laboratory, School of Electrical and Computer Engineering, University of

Tehran, Iran

*** Now: Beijing Laboratory for Electron Microscopy, Institute of Physics; Chinese Academy of

Sciences, China

**** Max Planck Institute for Solid State Research, Heisenbergstraße 1, D-70569 Stuttgart

It is not until recently that it has become possible to image localized surface plasmon resonances (LSPRs) with a spatial resolution in the nanometer-range over the entire wavelength range from far infrared up to the ultraviolet [1]. In particular the far infrared regime has become more readily accessible by the introduction of electron monochromators which provide an energy resolution below $100 \mathrm{meV}$. In our work we make use of an imaging energy filter which allows us to directly acquire images at selected energy-losses corresponding to LSPRs. So far this method has been applied on nanotriangles [2-4], nanorods [5], arrangements of circular nanoholes [6, 7], and nanoslits [8] to directly map the plasmonic eigenmodes. The experiments were carried out at the SESAM microscope (Zeiss, Oberkochen, Germany) operated at $200 \mathrm{kV}[9,10]$. We compare our experimental results with simulations based on the discrete-dipole approximation (DDA), 3D-Finite-difference time-domain (3DFDTD), and finite-element (FEM) methods. It will be demonstrated that the combination of experiment and simulation is important for a thorough understanding of the excitation and type of LSPRs.

As an example, FIG.1 shows energy-filtered images of resonant surface plasmon modes in a $\mathrm{Au}$ nanotriangle. These modes are characterized by equidistant field maxima along the edges of the nanotriangle. The separations of field maxima allow us to measure the plasmon dispersion (FIG.2). We found that this dispersion does neither correspond to a plasmon on a free Au surface nor to an interface plasmon between $\mathrm{Au}$ and $\mathrm{Si}_{3} \mathrm{~N}_{4}$ which was used as substrate. A comparison with simulation data will be presented which explains this unexpected plasmonic behaviour.

Further results will be shown highlighting the coupling effect of plasmonic fields in closely spaced nanoobjects.

References

[1] J. Nelayah et al., Nature Physics 3 (2007) 348.

[2] J. Nelayah et al., Opt. Lett. 34 (2009) 1003.

[3] J. Nelayah et al., Proceedings of the 14th European Microscopy Congress, S. Richter and A. Schwedt, eds. (Springer, 2008), Vol. 2, 243-244.

[4] L. Gu et al., submitted to Physical Review B (2011).

[5] B. Schaffer et al., Phys. Rev. B 79 (2009) 041401.

[6] W. Sigle et al., Opt. Lett. 34 (2009) 2150.

[7] W. Sigle et al., Ultramicroscopy 110 (2010) 1094.

[8] B. Öğüt et al., to be submitted.

[9] C.T. Koch et al., Microscopy and Microanalysis 12 (2006) 506.

[10] E. Essers et al., Ultramicroscopy 110 (2010) 971. 


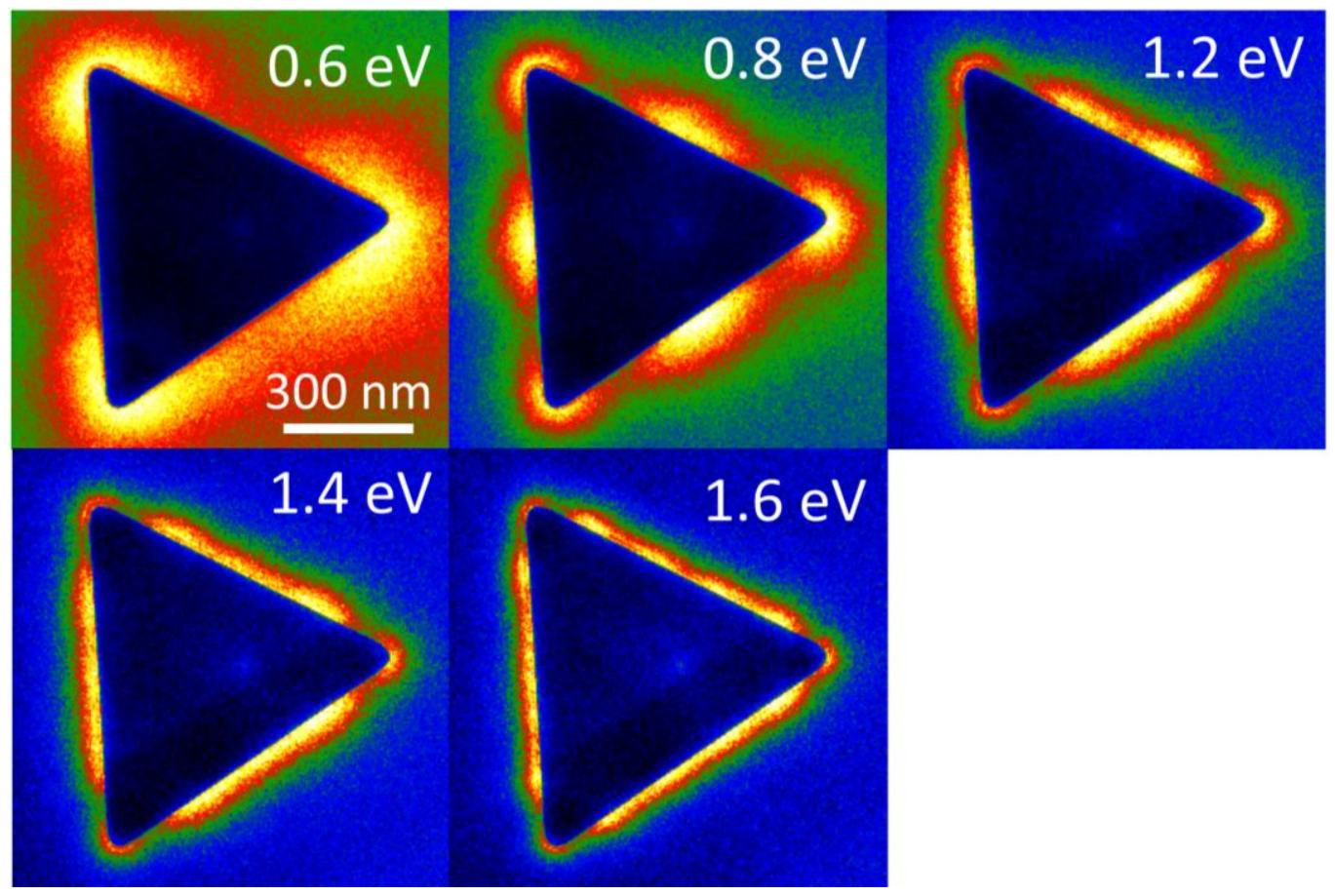

FIG.1: EFTEM images of a Au nanotriangle acquired at energy losses of $0.6 \mathrm{eV}, 0.8 \mathrm{eV}, 1.2 \mathrm{eV}, 1.4$ $\mathrm{eV}$, and $1.6 \mathrm{eV}$.

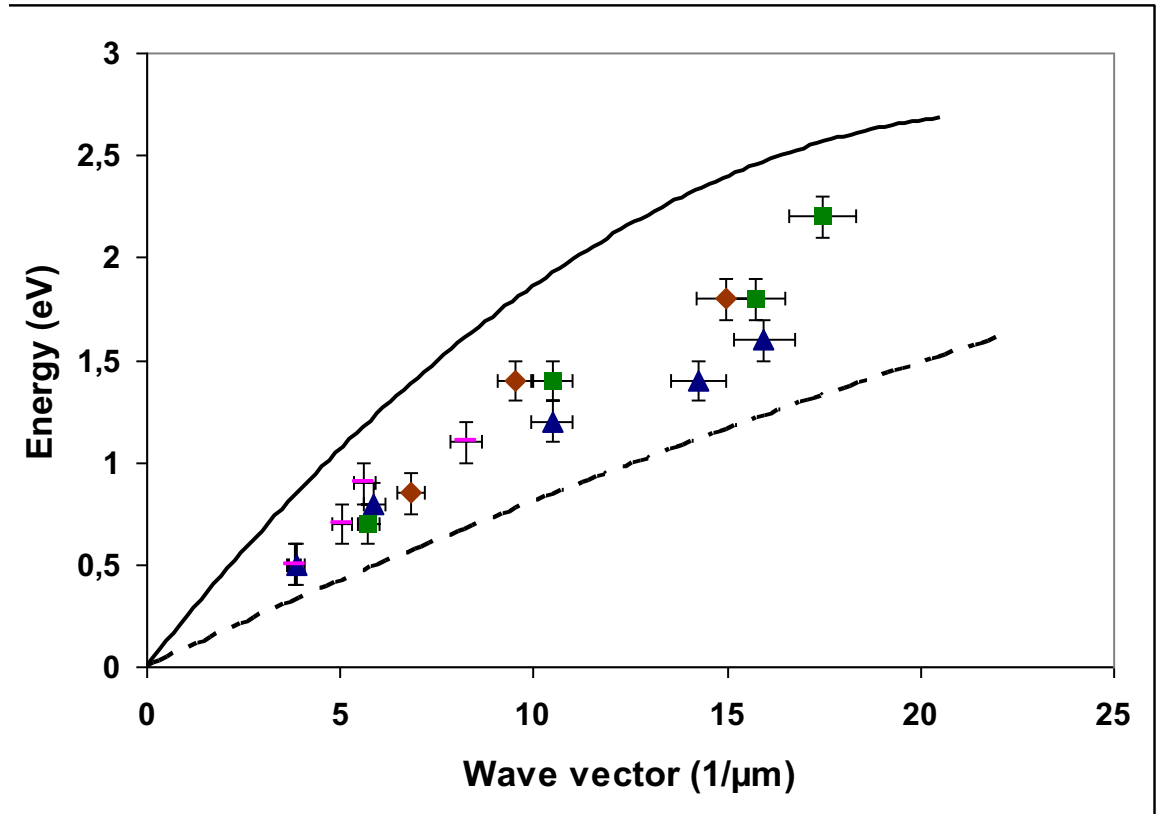

FIG.2: Plasmon dispersion extracted from the EFTEM data of triangles (blue), hexagons (brown, green), and rods (magenta). Plasmon dispersion curves for a free Au surface (bold line) and the interface between $\mathrm{Au}$ and $\mathrm{Si}_{3} \mathrm{~N}_{4}$ (dashed line) are shown for comparison. 